\title{
Clinical characteristics and outcomes of antenatal fetal intra-abdominal umbilical vein varix detection
}

\author{
Si Won Lee ${ }^{1^{*}}$, Moon Young Kim ${ }^{1}$, Jung Eun Kim ${ }^{1 *}$, Jin Hoon Chung ${ }^{1}$, Hyun Jung Lee ${ }^{2}$, Ji Young Yoon ${ }^{1}$ \\ Department of Obstetrics and Gynecology, ${ }^{1}$ Cheil General Hospital and Women's Healthcare Center, Kwandong University College of Medicine, Seoul; \\ ${ }^{2}$ Woosung Woman's Hospital, Ansan, Korea
}

\section{Objective}

This study reviewed clinical characteristics of fetal intra-abdominal umbilical vein (FIUV) varices that were detected during antenatal ultrasound examinations.

\section{Methods}

Between January 2006 and January 2012, 121 cases of FIUV varices were detected and 7 cases were lost to follow-up. We retrospectively reviewed the medical records of 114 patients and neonates.

\section{Results}

From a total 96,553 ultrasound examinations in 43,995 pregnancies, 121 cases of FIUV varices were identified ( 2.8 per 1,000 pregnancies). Gestational age at diagnosis was $32.0 \pm 2.9$ weeks (range, 20.1-36.3 weeks), the mean diameter of the FIUV varix was $12.6 \pm 2.1 \mathrm{~mm}$ (range, $8.0-21.0 \mathrm{~mm}$ ) at initial diagnosis and the mean maximal diameter was $13.1 \pm 2.3 \mathrm{~mm}$ (range, $8.0-21.0 \mathrm{~mm}$ ) during follow-up. The most severe pregnancy complications included one case of intrauterine fetal death and another case of fetal hydrops. Associated fetal anomalies $(n=11,9.6 \%)$ detected by ultrasonography included bilateral renal pelvis dilatation, ventriculomegaly, cryptorchidism, incomplete renal duplication and pulmonary sequestration. A total of 104 cases $(91.2 \%)$ were delivered at term and 10 cases (8.8\%) were preterm deliveries before 37 weeks of gestation.

\section{Conclusion}

FIUV varices that are not associated with fetal anomalies based on ultrasound examination during prenatal care have favorable pregnancy outcomes. Nevertheless, close fetal monitoring is recommended to decrease perinatal complications.

Keywords: Antenatal ultrasound; Umbilical veins; Varicose veins

\section{Introduction}

Fetal intra-abdominal umbilical vein (FIUV) varix is defined as focal dilatation of the umbilical venous diameter at the level of cord insertion. The diameter of the umbilical vein normally increases linearly with gestational age. However, although FIUV is rare and the cause is currently unknown, the condition can be diagnosed by antenatal ultrasound and only about 100 cases have been reported in the literature so far [1]. Ultrasound FIUV varix findings indicate intra-abdominal dilation, which is usually an extrahepatic portion of the umbilical vein. The weakest part of the umbilical circulation
Received: 2013.9.27. Revised: 2013.10.21. Accepted: 2013.10.22. Corresponding author: Moon Young Kim Department of Obstetrics and Gynecology, Cheil General Hospital and Women's Healthcare Center, 17 Seoae-ro 1-gil, Jung-gu, Seoul 100-380, Korea

Tel: +82-2-2000-7126 Fax: +82-2-2271-1734

E-mail: mykimdr@yahoo.co.kr

*These authors contributed equally to this study.

Articles published in Obstet Gynecol Sci are open-access, distributed under the terms of the Creative Commons Attribution Non-Commercial License (http://creativecommons. org/licenses/by-nc/3.0/) which permits unrestricted non-commercial use, distribution, and reproduction in any medium, provided the original work is properly cited.

Copyright ( $\odot 2014$ Korean Society of Obstetrics and Gynecology 


\section{Obstetrics \& Gynecology Science}

Vol. 57, No. 3, 2014
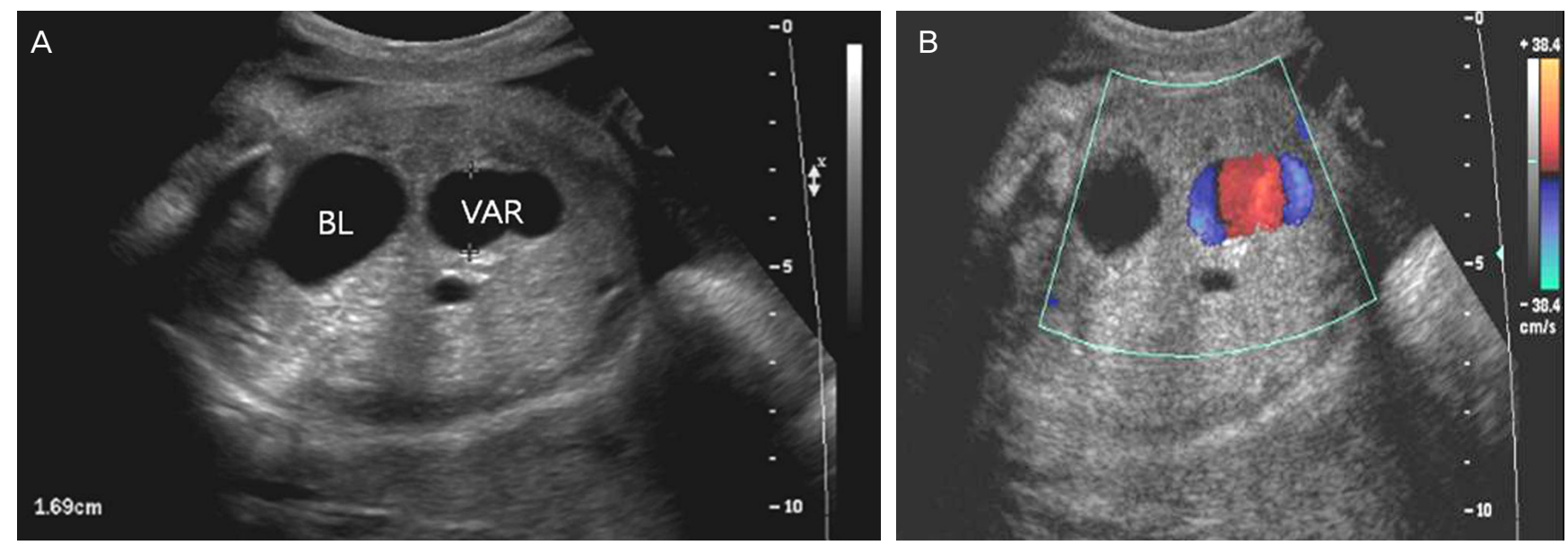

Fig. 1. (A) Transverse view of the lower fetal abdomen showing an umbilical vein varix that was approximately $16.9 \mathrm{~mm}$ at 33 weeks of gestation. (B) Color Doppler shows some turbulence in the intravascular area and differentiates from other cystic lesions. BL, bladder; VAR, fetal umbilical vein varix.

is the extrahepatic intra-abdominal portion of the umbilical circulation and therefore any condition that can increase the umbilical venous pressure could potentially induce umbilical vein dilation [2].

Color-flow Doppler can be helpful for diagnosing FIUV because it displays continuity with the umbilical vein and distinguishes vascular structure from other cystic lesions, for example the distended gallbladder or cysts in other structures. Diagnostic criteria reported by Nyberg [2] include an umbilical vein diameter more than $9 \mathrm{~mm}$ or a varix enlargement that is at least $50 \%$ more than the diameter of the intrahepatic umbilical vein. In nomograms of umbilical veins published by Weissman et al. [3], the average diameter of the umbilical vein varied from $2 \mathrm{~mm}$ at 14 to 15 weeks of gestation to 7 to $8 \mathrm{~mm}$ at term.

FIUV varix has been reported to be associated with intrauterine fetal death (IUFD), chromosomal abnormalities, fetal hydrops and other adverse pregnancy outcomes [4-6]. In this study, our objective was to evaluate clinical characteristics and outcomes of all fetuses with a FIUV varix at our institute.

\section{Materials and methods}

This was a retrospective case series study. All cases with a diagnosis of FIUV varix identified at Cheil General Hospital between January 2006 and January 2012 were reviewed using our ultrasound database. During the study period, a total of 96,553 ultrasound examinations were conducted in

\section{3,995 patients.}

Ultrasonography was performed using a convex abdominal transducer at a frequency of either 3.5 or $5 \mathrm{MHz}$ with one of the following ultrasound models (Voluson 730, GE, Milwaukee, WI, USA; LOGIQ 400 or 500, GE). Although all routine ultrasound examinations were evaluated for FIUV varices, measurement was performed only in cases with suspicious abnormal dilatation. In cases with a suspected FIUV varix, color-flow Doppler imaging and further high resolution ultrasonography for detailed fetal anatomic survey were taken for confirmation (Fig. 1).

The FIUV varix diameter was measured from one outer edge to the opposite inner edge with electrical calipers on axial images immediately cephalad to the insertion of the umbilical vein into the fetal abdomen. A FIUV varix was diagnosed based on previously reported diagnostic criteria by Nyberg [2] of a focal dilatation greater than $9 \mathrm{~mm}$, or at least $50 \%$ larger than the intrahepatic umbilical vein.

We reviewed the medical records of all cases with an FIUV varix diagnosis and clinical characteristics, the gestational age at first diagnosis, presence or absence of associated fetal anomaly, pregnancy outcomes, and postnatal ultrasonography findings of the neonates were recorded. Descriptive statistics were used and reported as mean \pm SD and range. Categorical data were analyzed using chi-square tests. A $P$-value less than 0.05 was considered statistically significant. This study was approved by the ethics committee of Cheil General Hospital and the Women's Healthcare Center. 


\section{Obstetrics \& Gynecology Science}

Si Won Lee, et al. Characteristics of fetal intra-abdominal umbilical vein varix

\section{Results}

A total of 96,553 ultrasound examinations were performed in 43,995 patients at our institution and we identified 121 cases of FIUV varices. The incidence rate was calculated to be

Table 1. Demographic characteristics of the study population $(n=114)$

\begin{tabular}{|lc|}
\hline Variables & Values \\
\hline Maternal age (yr) & $31.4 \pm 3.2(20-39)$ \\
$\geq 35$ & $19(16.7)$ \\
$<35$ & $95(83.3)$ \\
Proportion of gravity & $2.0 \pm 1.2(1-5)$ \\
Primigravida & $50(43.8)$ \\
Multigravida & $94(56.2)$ \\
Proportion of parity & $0.5 \pm 0.6(0-3)$ \\
Primipara & $67(58.8)$ \\
Multipara & $47(41.2)$ \\
Twin pregnancy & $6(5.3)$ \\
\hline
\end{tabular}

Values are presented as mean \pm SD (range) or number (\%). approximately 2.8 cases per 1,000 pregnancies. Seven cases were excluded from the analysis because the delivery did not occur at our institution and outcome data were not available. Therefore, a total of 114 mother-infant pairs were included in the study. Six cases (5.3\%) were twin pregnancies and all twin pregnancies were dichorionic-diamniotic and only one of the twins was affected. The demographic characteristics of the study population are shown in Table 1.

We categorized the study subjects into three groups: diagnosis before 24 weeks, diagnosis between 24 and 30 weeks and diagnosis after 30 weeks of gestation. More than twothirds $(83.3 \%)$ of cases were diagnosed after 30 weeks of gestation. The mean FIUV varix diameter at initial diagnosis was $12.6 \pm 2.1 \mathrm{~mm}$ (range, $8.0-21.0 \mathrm{~mm}$ ) and the mean maximal diameter was $13.1 \pm 2.3 \mathrm{~mm}$ (range, $8.0-21.0 \mathrm{~mm}$ ) during follow-up. Only eight of our cases showed more than a 2-mm increase in the FIUV varix diameter from initial diagnosis to delivery. Four of the eight cases were initially diagnosed before 24 weeks and the other four cases were diagnosed at $24+3$ weeks, 25 weeks, $29+3$ weeks and $32+3$ weeks of gestation, respectively. One hundred and three cases (90.4\%)

Table 2. FIUV varices and other ultrasound findings

\begin{tabular}{|c|c|}
\hline Variables & Values \\
\hline Gestational weeks at initial diagnosis (wk) & $32.0 \pm 2.9(20.1-36.3)$ \\
\hline$<24$ & $4(3.5)$ \\
\hline $24-30$ & $15(13.2)$ \\
\hline$\geq 30$ & $95(83.3)$ \\
\hline FIUV diameter at initial diagnosis (mm) & $12.6 \pm 2.1(8.0-21.0)$ \\
\hline Maximum diameter of FIUV (mm) & $13.1 \pm 2.3(8.0-21.0)$ \\
\hline Isolated FIUV varix & $103(90.4)$ \\
\hline Amniotic fluid index at initial diagnosis & $12.5 \pm 4.5(2.5-32.0)$ \\
\hline Overall cases with associated anomalies or pregnancy complications & $11(9.6)$ \\
\hline Hydrops fetalis & 1 \\
\hline Cryptorchidism & 2 \\
\hline Atrial septal defect & 1 \\
\hline Pulmonary sequestration & 1 \\
\hline Renal pelvis dilatation & 1 \\
\hline Incomplete unilateral duplex kidney & 1 \\
\hline Cerebral ventriculomegaly & 2 \\
\hline Non-lethal skeletal dysplasia & 1 \\
\hline Single umbilical artery & 1 \\
\hline
\end{tabular}

Values are presented as mean \pm SD (range) or number (\%) or number.

FIUV, fetal intra-abdominal umbilical vein varix. 


\title{
Obstetrics \& Gynecology Science
}

\author{
Vol. 57, No. 3, 2014
}

showed an isolated FIUV varix without associated anomalies. Eleven cases (9.6\%) had associated anomalies on ultrasound examination and there were no chromosomal abnormalities in the study population. Associated anomalies included hydrops fetalis, cryptorchidism, atrial septal defect, pulmonary sequestration, renal pelvis dilatation, incomplete unilateral duplex kidney, cerebral ventriculomegaly, non-lethal skeletal dysplasia and a single umbilical artery (Table 2).
Of the total number of cases, 104 (91.2\%) were delivered at term and 10 cases (8.8\%) were preterm deliveries before 37 weeks of gestation (Table 3). There was one case of IUFD in a 29-year-old mother at 40 weeks of gestation. The FIUV varix was diagnosed at 29 weeks in the IUFD case and was measured to be approximately $10 \mathrm{~mm}$ in diameter. Increased nuchal translucency of $4 \mathrm{~mm}$ in the first trimester ultrasound was also noted in this fetus and the fetal karyotype was nor-

Table 3. Pregnancy outcomes

\begin{tabular}{|c|c|}
\hline Variables & Values \\
\hline Gestational weeks at delivery (wk) & $38.9 \pm 1.6(32.3-41.1)$ \\
\hline $28-33(+6)$ & $2(1.8)$ \\
\hline $34-36(+6)$ & $8(7.0)$ \\
\hline Full term delivery & $104(91.2)$ \\
\hline \multicolumn{2}{|l|}{ Mode of delivery } \\
\hline Normal vaginal delivery & $72(63.2)$ \\
\hline Elective cesarean section & $27(23.7)$ \\
\hline Emergency cesarean section & $13(11.4)$ \\
\hline Vacuum delivery & $2(1.8)$ \\
\hline Induction & $9(7.9)$ \\
\hline Karyotype abnormality & $0(0)$ \\
\hline Birth weight (g) & $3,226.0 \pm 459.5(2,020-4,250)$ \\
\hline \multicolumn{2}{|l|}{ Neonate gender } \\
\hline Male & $50(43.9)$ \\
\hline Female & $64(56.1)$ \\
\hline Apgar score $1 \mathrm{~min}$ & $8.2 \pm 0.9(3-9)$ \\
\hline Apgar score 5 min & $8.9 \pm 0.6(6-10)$ \\
\hline NICU admission & $8(7.0)$ \\
\hline \multicolumn{2}{|l|}{ Delivered at 40 wk: GER } \\
\hline \multicolumn{2}{|l|}{ Delivered at 39 wk: pneumothorax } \\
\hline \multicolumn{2}{|l|}{ Delivered at 33 wk: RDS, anemia, septicemia } \\
\hline \multicolumn{2}{|c|}{ Delivered at 38 wk: neonatal jaundice, multiple muscular VSD } \\
\hline \multicolumn{2}{|l|}{ Delivered at 36 wk: pneumonia } \\
\hline \multicolumn{2}{|c|}{ Delivered at 40 wk: pulmonary sequestration, respiratory distress } \\
\hline \multicolumn{2}{|c|}{ Delivered at 41 wk: meconium aspiration syndrome } \\
\hline \multicolumn{2}{|c|}{ Delivered at 40 wk: respiratory distress syndrome } \\
\hline Overall cases with pregnancy complications & $17(14.9)$ \\
\hline Intrauterine fetal death & $1(0.9)$ \\
\hline Oligohydramnios (AFI <5 cm) & $6(5.3)$ \\
\hline Intrauterine fetal growth restriction & $4(3.5)$ \\
\hline Preeclampsia & $1(0.9)$ \\
\hline Gestational diabetes mellitus & $4(3.5)$ \\
\hline Placental previa & $1(0.9)$ \\
\hline
\end{tabular}

Values are presented as mean \pm SD (range) or number (\%).

NICU, neonatal intensive care unit; GER, gastroesophageal reflux; RDS, respiratory distress syndrome; VSD, ventricular septal defect; AFI, amniotic fluid index. 


\section{Obstetrics \& Gynecology Science}

Si Won Lee, et al. Characteristics of fetal intra-abdominal umbilical vein varix

mal. There were no associated anomalies on mid-trimester ultrasound examination. There were six twin pregnancies, all were dichorionic-diamniotic, and only one of the fetuses was affected in each twin pair. All twin cases were delivered after 35 weeks of gestation and all the affected babies were healthy. The rate of cesarean section in our study population was $35.1 \%$, which was not significantly different compared to the total cesarean section rate (36.3\%) at our institution during the study period $(P=0.842)$. The rate of emergency cesarean section due to fetal distress or failure to progress and induction of labor was not increased and the mode of delivery was not affected by the presence of the disease.

There were 8 cases $(7.0 \%)$ of neonatal intensive care unit (NICU) admission. The reasons for NICU admission were gastro-esophageal reflux, pneumothorax, respiratory distress syndrome and septicemia due to preterm delivery, neonatal jaundice and ventricular septal defect (multiple muscular types), pneumonia, pulmonary sequestration, meconium aspiration syndrome and respiratory distress syndrome. Other pregnancy complications were noted in 17 cases (14.9\%) and the most common complications included oligohydramnios $(5.3 \%)$, intrauterine growth restriction (3.5\%) and gestational diabetes mellitus (3.5\%) (Table 3).

\section{Discussion}

FIUV varix is considered to be a developed lesion rather than a congenital anomaly because more than two-thirds of the cases are diagnosed after 30 weeks of gestation. As the incidence of this condition is very low, the clinical significance is controversial. Only case reports and case series of this condition have been discussed and the largest case series, which was reported by Byers et al. [7], evaluated the characteristics of 52 cases of FIUV varices. Their study found an association between FIUV varices and chromosomal anomalies (3 cases of trisomy 21, 5.8\%) and other structural anomalies $(28.8 \%)$. Compared to other previous reports, this rate of fetal anomaly was slightly lower, but the rate of chromosomal anomaly was significantly higher. Therefore, Byers et al. [7] suggested that FIUV varix could be a fetal aneuploidy marker.

Weissmann-Brenner et al. [8] recently summarized 109 cases of FIUV varices from several different papers and found that the prognosis of 'isolated' FIUV varix or those with asso- ciated anomalies were both fairly good. In the study, $62.4 \%$ of the cases were full-term deliveries with normal pregnancy outcomes, $11 \%$ were preterm deliveries with normal pregnancy outcomes, $2.7 \%$ involved postnatal complications and $13.7 \%$ resulted in IUFD including 5 cases with chromosomal or structural abnormalities.

In this study we evaluated 114 cases of FIUV varices with favorable pregnancy outcomes. Some previous reports showed that the outcome of a FIUV varix diagnosed before 26 weeks of gestation was worse than FIUV varices that were diagnosed later in pregnancy $[5,9]$. The reports found that the rates of IUFD, umbilical vein thrombosis and non-reassuring fetal heart rate monitoring were higher when a FIUV varix was diagnosed earlier in pregnancy. Only eight of our cases showed more than a 2-mm increase in the FIUV varix diameter from initial diagnosis to delivery before 33 weeks of gestation, respectively, implying that earlier diagnosis of this condition is associated with size increment and possibly poorer pregnancy outcomes. However, we could not identify a correlation between the change in size and poor maternal or neonatal outcomes.

Currently, there are no defined guidelines for managing pregnancies with FIUV varices. Some authors recommend inducing labor after confirmation of fetal lung maturity or when there are signs of fetal compromise [10] and others recommend weekly follow-up with Doppler from diagnosis to 28 weeks of gestation and twice a week afterwards [8].

In our institute, once FIUV varix was suspected, high-resolution ultrasound with Doppler was performed to assess fetal structural anomalies and the patients were followed weekly with electronic fetal monitoring until delivery. If a diagnosis of FIUV varix was made before 32 weeks of gestation, followup ultrasound was performed between 32 and 36 weeks of gestation. If there were any sign of fetal compromise, labor was induced or a cesarean section was performed. However, we did not induce labor earlier than is usually recommended based only on the FIUV varix diagnosis or the size of the varix. Unfortunately, in spite of close monitoring, we had one case of IUFD one day after hospital follow-up.

There were 8 cases (7.0\%) of NICU admission and 5 cases were associated with respiratory problems. One case with pulmonary sequestration was transferred to a tertiary center for vascular surgery.

In this study, we identified 102 cases of isolated FIUV varices and all of these cases showed good neonatal outcomes 


\title{
Obstetrics \& Gynecology Science
}

\author{
Vol. 57, No. 3, 2014
}

without any IUFD. One previous report reviewed several literature publications and their analysis reported 5 cases of IUFD from 62 isolated FIUV varices [9]. Our only case of IUFD had a normal karyotype, but showed increased nuchal translucency up to $4 \mathrm{~mm}$ in the first trimester. Therefore, we classified this case as non-isolated.

This study is one of the largest case series on FIUV varices that has been reported to date. Although the general outcome of this condition is favorable, we had one case of IUFD despite close monitoring in our case series. In addition, previous case series and reports also showed some cases of IUFD despite meticulous examination and monitoring of the fetuses. Therefore when a diagnosis of FIUV is made on routine ultrasound examination, we suggest targeted ultrasound to rule out the possibility of other associated anomalies. If there are additional sonographic abnormalities, fetal karyotyping should be performed and follow-up ultrasound should be offered to look for size changes in the varix or additional abnormalities. Even if the FIUV varix is isolated, meticulous fetal monitoring should be recommended from the time of diagnosis at least once a week until delivery. Currently, the timing of delivery is very controversial based on previous reports. The results of our case series indicated that good outcomes were associated with most patients with isolated FIUV varices. Therefore when there are no co-existing abnormalities, we recommend that physicians follow routine protocol for delivery and consider inducing labor once the patient has reached 39 to 40 weeks of gestation.

This case series was limited because of the retrospective study design and because serial Doppler testing was not used to watch for turbulent flow. Therefore, additional, larger prospective studies should be conducted in the future. Additional studies can work to validate the significance of FIUV varices and determine the best patient management strategies.

\section{Conflict of interest}

No potential conflict of interest relevant to this article was reported.

\section{References}

1. Mantas N, Sifakis S, Koukoura O, Avgoustinakis E, Koumantakis E. Intraabdominal umbilical vein dilatation and term delivery: a case report and review of the literature. Fetal Diagn Ther 2007;22:431-4.

2. Nyberg D. Varix of the umbilical vein. In: Nyberg DA, McGahan JP, Pretorius DH, Pilu G, editors. Diagnostic imaging of fetal anomalies. Philadelphia: Lippincott Williams \&Wilkins; 2003. p.114-5.

3. Weissman A, Jakobi P, Bronshtein M, Goldstein I. Sonographic measurements of the umbilical cord and vessels during normal pregnancies. J Ultrasound Med 1994;13:11-4.

4. Mahony BS, McGahan JP, Nyberg DA, Reisner DP. Varix of the fetal intra-abdominal umbilical vein: comparison with normal. J Ultrasound Med 1992;11:73-6.

5. Sepulveda W, Mackenna A, Sanchez J, Corral E, Carstens E. Fetal prognosis in varix of the intrafetal umbilical vein. J Ultrasound Med 1998;17:171-5.

6. Rahemtullah A, Lieberman E, Benson C, Norton ME. Outcome of pregnancy after prenatal diagnosis of umbilical vein varix. J Ultrasound Med 2001;20:135-9.

7. Byers BD, Goharkhay N, Mateus J, Ward KK, Munn MB, Wen TS. Pregnancy outcome after ultrasound diagnosis of fetal intra-abdominal umbilical vein varix. Ultrasound Obstet Gynecol 2009;33:282-6.

8. Weissmann-Brenner A, Simchen MJ, Moran O, Kassif $E$, Achiron $R$, Zalel $Y$. Isolated fetal umbilical vein varix: prenatal sonographic diagnosis and suggested management. Prenat Diagn 2009;29:229-33.

9. Fung $T Y$, Leung TN, Leung TY, Lau TK. Fetal intra-abdominal umbilical vein varix: what is the clinical significance? Ultrasound Obstet Gynecol 2005;25:149-54.

10. Zalel Y, Lehavi O, Heifetz S, Aizenstein O, Dolitzki M, Lipitz $S$, et al. Varix of the fetal intra-abdominal umbilical vein: prenatal sonographic diagnosis and suggested in utero management. Ultrasound Obstet Gynecol 2000;16:476-8. 